\title{
Theory of Vector-Valued Hyperfunctions
}

\author{
By
}

\author{
Patrick D. F. IoN* and Takahiro KAWAI
}

\section{Contents}

\author{
§. Introduction. \\ $\S 1$. Definition and properties of the sheaves ${ }^{E} \mathcal{O}$ and ${ }^{E} \mathscr{E}$. \\ $\S 2$. Pure codimensionality of $\boldsymbol{R}^{n}$ with respect to ${ }^{E_{\mathcal{O}}}$. \\ $\S 3$. Hyperfunctions as boundary values. \\ $\S 4$. Hyperfunctions with holomorphic parameters.
}

\section{§. Introduction}

The theory of hyperfunctions has now been flourishing for some years since its conception by M. Sato [Sato 1959/60 and earlier references therein]. It is a natural enough exercise to try to extend the theory and its developments to cover the cases of hyperfunctions and the like with value in a locally convex (topological vector) space, as has been done for distributions by their originator L. Schwartz [Schwartz 1957, 1958]. Indeed this is also required for applications [e.g. Ouchi 1972 for the one variable case, Ion in prep.]. The purpose of this note is to point out that a start may easily be made using the methods of 'soft analysis', to define the sheaf ${ }^{E} \mathscr{B}$ of $E$-valued hyperfunctions for a Fréchet space $E$ and to explore the basic properties of $E \mathscr{B}$.

The methods employed in this note will be used to discuss $E$-valued microfunctions in our subsequent papers.

Section 1 contains the facts which need to be recalled concerning the Dolbeault resolution, and three theorems of Grothendieck on tensor products which essentially make the proofs go. We also remark that Palamodov's result on the splitting of the Dolbeault complex over a

Received December 18, 1973.

* Universität Heidelberg. Work done while visiting the Research Institute for Mathematical Sciences, Kyoto University. 
Stein manifold extends to the vector-valued case.

Section 2 contains the required discussion of the pure codimensionality of $\boldsymbol{R}^{n}$, which involves a simple generalization of the Malgrange Vanishing Theorem, and the resulting definition of $E \mathscr{B}$.

Section 3 contains a discussion of expressing hyperfunctions as boundary values of holomorphic functions and section 4 a discussion of hyperfunctions with holomorphic parameters.

References are cited in the form: author followed by the last two digits of the year of publication.

\section{Acknowledgement}

The first named author (P. D. F. I.) would like to thank the Research Institute for Mathematical Sciences for the generous hospitality shown him, and the Royal Siciety for the fellowship under the Royal Society Japan Programme that made his stay there possible.

The second named author (T. K.) would like to thank the Sakkokai Foundation for the partial support generously afforded him during the preparation of this work.

\section{§1. Preliminaries}

Throughout the paper $E$ will denote a quasi-complete separated locally convex topological vector space. For a discussion of the reasons for assuming quasi-completeness see [Schwartz 52]. We shall denote by $M$ a given real analytic manifold with $\operatorname{dim}_{R} M=m$ and by $X$ its complexification, so that $\operatorname{dim}_{C} X=m$. Let us begin by defining our basic function sheaves.

Definition 1.1. Denote by ${ }^{E} \mathscr{E}$ the sheaf of germs of infinitely differentiable (=smooth) functions on $X$ with values in the vector space $E$. The space $\Gamma\left(S,{ }^{E} \mathscr{E}\right) \equiv{ }^{E} \mathscr{E}(S)$ of sections over an open set $S$ will be endowed with the usual topology of uniform convergence of all derivatives on compact subsets.

Recall that the definition of smooth $E$-valued functions given by 
Schwartz as $\mathscr{E}(U) \varepsilon E=\mathscr{E}(U, E)$ for $U$ open, for $E$ quasi-complete and separated, is equivalent to the definition $\mathscr{E}(U, E)=\mathscr{E}(U) \otimes_{\varepsilon} E$ [Schwartz 52 Thm. 1], which for a complete $E$ is in fact $\mathscr{E}(U) \widehat{\otimes}_{\varepsilon} E=\mathscr{E}(U) \hat{\otimes} E$ since $\mathscr{E}(U)$ is nuclear. [ibid. and Treves 67 Thm. 51.5 Cor.]

Definition 1.2. Denote by ${ }^{E} \mathcal{O}$ the sheaf of germs of holomorphic functions on $X$ with values in the vector space $E$. This ${ }^{E} \mathcal{O}$ is a subsheaf of ${ }^{E} \mathscr{E}$ and the space of sections $\Gamma\left(U,{ }^{E} \mathcal{O}\right) \equiv{ }^{E} \mathcal{O}(U)$ will be given the topology induced from that of $E_{\mathscr{E}}(U)$.

Recall that the definition of holomorphy for a vector-valued function can be given in terms of the Cauchy integral (starting from a function known to be continuous), or by the Cauchy-Riemann equations (starting from a function known to have partial derivatives, or in particular thought of as defining a subsheaf of ${ }^{E} \mathscr{E}$ ), or as weak holomorphy. Furthermore these definitions are all equivalent (on the intersection of their domains) if the space $E$ is quasi-complete, so that the closed convex hull of a compact set in $E$ is also compact [Grothendieck 52].

A property basic for cohomology theory is the following.

Proposition 1.3. The sheaf ${ }^{E} \mathscr{E}$ is soft.

Proof. This is an immediate consequence of the fact that ${ }^{E} \mathscr{E}$ is fine (admits partitions of unity subordinate to it), which, since $E_{\mathscr{E}}$ is obviously an $\mathscr{E}$-module, is so for exactly the same reason that $\mathscr{E}$ is fine [Gunning and Rossi 65 Chap. VI. A].

Next we remark the exactness of the Dolbeault complex, which, as a consequence of Prop. 1.3, we now know will give a soft resolution of $E_{\mathcal{O}}$.

First we must introduce, for non-negative integers $p$ and $q$, the sheaves ${ }^{E} \mathscr{E}^{(p, q)}$ of germs of $E$-valued differential forms of type $(p, q)$; a typical element of ${ }^{E} \mathscr{E}^{(p, q)}(U)$ for any sufficiently small set $U$ open in $X$ is, in complex local coordinates $\left(z_{1}, \ldots, z_{m}\right)$

$$
\Sigma f_{I, J} d z_{I} \wedge d \bar{z}_{J}
$$


where the sum is over all disjoint ordered subsets of $(1, \ldots, m), I=\left(i_{1}\right.$, $\left.\ldots, i_{p}\right)$ and $J=\left(j_{1}, \ldots, j_{q}\right)$ with respectively $p$ and $q$ elements, and the $f_{I, J}$ are in ${ }^{E} \mathscr{E}(U)$, and

$$
\begin{aligned}
& d z_{I}=d z_{i_{1}} \wedge \cdots \wedge d z_{i_{p}} \\
& d \bar{z}_{J}=d \bar{z}_{j_{1}} \wedge \cdots \wedge d \bar{z}_{j_{q}}
\end{aligned}
$$

if $p$ or $q$ be zero the corresponding index $I$ or $J$ and form $d z_{I}$ or $d \bar{z}_{J}$ are omitted, so ${ }^{E} \mathscr{E}^{(0,0)}={ }^{E} \mathscr{E}$.

Each sheaf $E_{\mathscr{E}^{(p, q)}}$, which is locally free of dimension $\left(\begin{array}{c}m \\ p\end{array}\right)\left(\begin{array}{c}m \\ q\end{array}\right)$ over ${ }^{E} \mathscr{E}$, is soft since ${ }^{E} \mathscr{E}$ is. Therefore the following is a soft (in fact, a fine) resolution of $E_{\mathcal{O}}$

$0 \longrightarrow{ }^{E} \mathcal{O} \longrightarrow{ }^{E} \mathscr{E}(0,0) \stackrel{\bar{\partial}_{0}}{\longrightarrow} E \mathscr{E}^{(0,1)} \stackrel{\bar{\partial}_{1}}{\longrightarrow} \cdots \stackrel{\bar{\partial}_{n-1}}{\longrightarrow} E_{\mathscr{E}}(0, n) \stackrel{\bar{o}_{n}}{\longrightarrow} 0$,

where locally

$$
\bar{\partial}_{q}\left(f_{J} d \bar{z}_{J}\right) \equiv \sum_{j=1}^{m}\left(\partial f_{J} / \partial \bar{z}_{j}\right) d \bar{z}_{j} \wedge d \bar{z}_{J}
$$

A fundamental property is that this Dolbeault resolution is exact.

Proposition 1.4. The complex $\left({ }^{E} \mathscr{E}^{(0, \cdot)}, \bar{\partial}\right)$ is exact, when $E$ is a quasi-complete separated locally convex space.

Proof. The proof of this fact goes exactly like that for the case $E=C$ via Cauchy's integral formula, since $E$ being quasi-complete, it has the compact convex closure property previously mentioned. Following Gunning and Rossi, it goes this way.

The assertion is a local one so that we may work without loss of generality in a neighbourhood of $z$ biholomorphically equivalent to a connected open set in $\mathbb{C}^{m}$.

Consider a point $z$ in $D$. We tackle the first node first. Consider a germ $\underline{f}$ in $E_{\mathscr{E}_{z}}^{(0,0)}$ and suppose $\bar{\partial}_{0} f=0$. Then there exists a smooth function $f$ defined in some open neighbourhood of $z$ in $D$, such that $f$ represents $\underline{f}$ and $\bar{\partial} f=0$. Then $f$, since it satisfies the Cauchy-Riemann equations, is holomorphic; so $\underline{f}$ in fact lies in the stalk $E_{\mathcal{O}_{z}}$. Therefore the kernel of the sheaf homomorphism $\bar{\partial}_{0}$ is exactly the subsheaf 
${ }^{E} \mathcal{O} \subset{ }^{E} \mathscr{E}$

Next consider $q>0$ and a germ $\underline{\varphi}$ in $E_{\mathscr{E}_{z}(0, q)}$, and select some smooth differential $(0, q)$-form $\varphi$, in ${ }^{E} \mathscr{E}^{(0, q)}(U)$ for some open neighbourhood $U$ of $z$, which represents $\underline{\varphi}$. If $U$ is sufficiently small then $\bar{\partial}_{q} \underline{\varphi}=0$ entails $\bar{\partial}_{q} \varphi=0$. By the following Dolbeault-Grothendieck lemma, which is where Cauchy's integral comes in, on a sufficiently small neighbourhood $U$ there is a smooth differential form $\psi$ in $E_{\mathscr{E}^{(}(0, q-1)}(U)$ such that $\bar{\partial}_{q-1} \psi=\varphi$. If we put $\Psi$ for the germ of $\psi$ at $z$ then $\bar{\partial}_{q-1} \Psi=\underline{\varphi}$. Since we are dealing with a complex, so that $\bar{\partial}_{q} \bar{\partial}_{q-1}=0$, this proves exactness.

\section{Lemma 1.6. (Dolbeault-Grothendicck)}

Let $D=D_{1} \times \cdots \times D_{m}$ be a bounded open polydisc in $C^{m}$, and let $\omega$ be a smooth E-valued differential form of bidegree $(q, p)$ in an open neighbourhood $U$ of $\bar{D}$. If $q>0$ and if $\overline{\hat{c}} \omega=0$, then there is a smooth differential form $\eta$ of bidegree $(p, q-1)$ in $D$ such that $\omega=\bar{\partial} \eta$.

Proof. [Hörmander 66 Theorem 2.3.3]

[Gunning and Rossi 65 Theorem I.D.3]

In terms of some coordinates $z=\left(z_{1}, \ldots, z_{m}\right), \omega$ may be written

$$
\omega=\sum_{I, J} w_{I, J} d z_{I} \wedge d \bar{z}_{J}
$$

where the coefficients $w_{I, J}$ are functions in $E_{\mathscr{E}}(U)$.

One proceeds through induction on the number counting from the left of the differentials $d \bar{z}_{1}, \ldots, d \bar{z}_{m}$, that may possibly be involved in the above explicit expression of $\omega$. Assume the first $k$ from the left are involved. We have to prove the assertion for $k=m$. The assertion for $k=0$ is trivially true, for if the expression of a form $\omega$ of bidegree $(p, q)$, with $q>0$, does not need to involve any differentials $d \bar{z}_{j}$, then $\omega$ is identically zero and $\bar{\partial} 0=0$ is also in $E_{\mathscr{E}}(p, q-1)(D)$.

Assume then $k \geqq 1$; in fact of course $k \geqq q$ if $\omega$ is not zero. Write

$$
\omega=d \bar{z}_{k} \wedge \alpha+\beta
$$

where the forms $\alpha$ and $\beta$ involve only $d \bar{z}_{1}, \ldots, d \bar{z}_{k-1}$. Now the assumption is 


$$
0=\bar{\partial} \omega=\left(d \bar{z}_{k} \wedge \bar{\partial} \alpha\right)+\bar{\partial} \beta
$$

Writing

$$
\begin{aligned}
& \alpha=\Sigma_{K, L} a_{K, L} d z_{K} \wedge d \bar{z}_{L} \\
& \beta=\Sigma_{K, L} b_{K, L} d z_{K} \wedge d \bar{z}_{L}
\end{aligned}
$$

we see that to satisfy the equation we must have

$$
\partial a_{K, L} / \partial \bar{z}_{j}=0=\partial b_{K, L} / \partial \bar{z}_{j}
$$

for $j>k$; that is the coefficients $a_{K, L}$ and $b_{K, L}$ are all holomorphic in the variables $z_{k+1}, \ldots, z_{m}$.

Consider any one of the coefficients $a_{K, L}$ and call it $f$ for short. This $f$ is an $E$-valued smooth function of each variable $z_{j}$ in an open neighbourhood $U_{j}$ of $\bar{D}_{j}$ and is in fact holomorphic in $z_{k+1}, \ldots, z_{m}$. There is then a function $g$ smooth in $U_{j}$ for $j \neq k$ and holomorphic there for $j>k$, and in addition smooth in some open neighbourhood $U_{k}^{\prime}$ of $\bar{D}_{k}$ which is contained in $U$, and such that $\partial g / \partial \bar{z}_{k}=f$.

The function $g$ may be constructed as follows using Cauchy's formula. Set $u=\left(z_{1}, \ldots, z_{k-1}\right)$ and $v=\left(z_{k+1}, \ldots, z_{m}\right)$ so that $z=\left(u, z_{k}, v\right)$.

Put

$$
g_{1}(z)=g_{1}\left(u, z_{k}, v\right) \equiv(2 \pi i)^{-1} \int_{D_{k}} f(u, \zeta, v)\left(\zeta-z_{k}\right)^{-1} d \zeta \wedge d \bar{\zeta}
$$

This function is obviously smooth in the parameters $u$ and $v$ and holomorphic in $v$. This function is however not necessarily holomorphic in $z_{k}$ where required. Consider therefore a smoothing function $\psi$ in $C^{\infty}\left(U_{k}\right)$ such that $\psi(\zeta)=1$ in the neighbourhood $U_{k}^{\prime}$ of $\bar{D}_{k}$ and $\psi(\zeta)=0$ outside a disc $D_{k}^{\prime}$ containing $U_{k}^{\prime}$ and contained in $U_{k}$. Define

$$
\begin{aligned}
g\left(u, z_{k}, v\right) & \equiv(2 \pi i)^{-1} \int_{D_{k}^{\prime}} f(u, \zeta, v) \psi(\zeta)\left(\zeta-z_{k}\right)^{-1} d \zeta \wedge d \bar{\zeta} \\
& =-(2 \pi i)^{-1} \int f\left(u, z_{k}-\zeta, v\right) \psi\left(z_{k}-\zeta\right) \zeta^{-1} d \zeta \wedge d \bar{\zeta}
\end{aligned}
$$

It is now clear that $g$ is smooth for $z$ in $D$. Since $\zeta^{-1}$ is integrable on any compact set, one may differentiate under the integral sign so that

$$
\partial g / \partial \bar{z}_{k}=-(2 \pi i)^{-1} \int \partial\left(f\left(u, z_{k}-\zeta, v\right) \psi\left(z_{k}-\zeta\right)\right) / \partial \bar{z}_{k} \zeta^{-1} d \zeta \wedge d \bar{\zeta}
$$




$$
\begin{aligned}
& =(2 \pi i)^{-1} \int_{D_{k}^{\prime}} \partial(f(u, \zeta, v) \psi(\zeta)) / \partial \bar{\zeta}\left(\zeta-z_{h}\right)^{-1} d \zeta \wedge d \bar{\zeta} \\
& =f\left(u, z_{k}, v\right) \psi\left(z_{k}\right)-(2 \pi i)^{-1} \int_{\partial D_{k}^{\prime}} f(u, \zeta, v) \psi(\zeta)\left(\zeta-z_{k}\right)^{-1} d \zeta \\
& =f\left(u, z_{k}, v\right),
\end{aligned}
$$

using Cauchy's integral formula, which is still valid for $E$-valued functions. The last equality follows from the fact that $\psi\left(z_{k}\right)=1$ and that $\psi(\zeta)=0$ on $\partial D_{k}^{\prime}$. Thus the required function $g$ has been explicitly constructed.

Replacing each of the coefficients $f=a_{K, L}$ by the corresponding $g$ $=c_{K, L}$ as constructed in the last paragraph one gets a new differential form

$$
\gamma=\Sigma_{K, L} c_{K, L} d z_{K} \wedge d \bar{z}_{L}
$$

such that

$$
\bar{\partial} \gamma=d \bar{z}_{h} \wedge \alpha+\delta
$$

where the remainder term $\delta$ involves only $d \bar{z}_{1}, \ldots, d \bar{z}_{k-1}$.

Consider now the form

$$
\varphi=\omega-\partial \gamma=\beta-\delta
$$

which satisfies $\bar{\partial} \varphi=0$, and note that it involves only the differentials $d \bar{z}_{1}, \ldots, d \bar{z}_{k-1}$. By the induction hypothesis there is a form $\chi$ such that $\varphi=\bar{\partial} \chi$, which concludes the proof since

$$
\omega=\bar{\partial}(\gamma+\chi)
$$

When the space $E$ is Fréchet, the use of the Cauchy integral formula may be passed over by using the general tensor product properties given as Theorems 1.8, 1.9 and 1.10. However, an even more informative result: the vector-valued generalization of a result of Palamodov holds [Palamodov 72].

Theorem 1.7. If $X$ is a Stein manifold and $E$ is a quasi-complete separated locally convex space then the operator $\bar{\partial}_{p}$ in the Dolbeault 
complex ${ }^{E} \mathscr{E}^{(0, p)}(X)$ splits for all $p>0$, that is $\bar{\partial}_{p}$ has a right inverse on its image. Hence for $p \geqq 2$

$$
H^{p}\left(X,{ }^{E} \mathcal{O}\right)=0
$$

Proof. We are again looking at the Dolbeault complex ${ }^{E} \mathscr{E}^{(0, \cdot)}(X)$ which, as has already been noted, is isomorphic to $\mathscr{E}^{(0, \cdot)}(X) \otimes E$; the operator $\bar{\partial}_{p}$ is of the form $\bar{\partial}_{p}^{c} \otimes 1$ where the $\bar{\partial}_{p}^{c}$ is the scalar one. Palamodov's Prop. 5.1 (loc. cit.) shows that $\bar{\partial}_{p}^{C}$ splits for $p>0$ and does not split for $p=0$. Now the splitting of $\bar{\partial}_{p}^{c}$ means that there is a right inverse $\rho$ for the first $\operatorname{map}\left(\bar{\partial}_{p}^{c}\right)^{2}$ in

$$
\mathscr{E}^{(0, p)} \longrightarrow \operatorname{im}_{\bar{p}}^{C} \hookrightarrow \mathscr{E}^{(0, p+1)},
$$

so that

$$
\left.\left(\bar{\partial}_{p}^{C}\right)^{`} \rho=\mathrm{id}_{(\mathrm{im} \bar{\partial}} C_{p}\right) .
$$

But then

$$
\begin{array}{r}
\left(\bar{\partial}_{\boldsymbol{p}}^{\boldsymbol{C}} \otimes 1\right)(\rho \otimes 1) \mid \operatorname{im}\left(\bar{\partial}_{\boldsymbol{\rho}}^{\boldsymbol{C}} \otimes 1\right) \\
=\left(\bar{\partial}_{\boldsymbol{p}}^{\boldsymbol{c}} \otimes 1\right)(\rho \otimes 1)=\mathrm{id} .,
\end{array}
$$

so that by extension from an equality holding for continuous operators on a dense subspace

$$
(\bar{\partial} \boldsymbol{c} \otimes 1)(\rho \otimes 1)=\mathrm{id}_{(\mathrm{im} \overline{\hat{o}}} \boldsymbol{C}_{\bar{p}} \hat{\otimes}_{1)}
$$

so that $\bar{\partial}_{p}^{\check{r}}$ has a right inverse too. Thus $\bar{\partial}_{p}$ splits whenever $\bar{\partial}_{p}^{C}$ does.

Theorem 1.8. Let, for $i=1,2, u_{i}: E_{i} \rightarrow F_{i}$ be continuous linear maps of locally convex spaces such that $u_{i} E_{i}$ is dense in $F_{i}$. Suppose additionally that $u_{1}$ and $u_{2}$ are open maps onto the images, that is that they are topological homomorphisms (homomorphisms of the category of locally convex spaces).

Then $u_{1} \hat{\otimes} u_{2}: E_{1} \hat{\otimes} E_{2} \rightarrow F_{1} \hat{\otimes} F_{2}$ is a topological homomorphism of the projectively completed tensor product $E_{1} \hat{\otimes} E_{2}$ onto a dense subspace of $F_{1} \hat{\otimes} F_{2}$. Further, if $E_{1}$ and $E_{2}$ are metrizable then $u_{1} \hat{\otimes} u_{2}$ is actually a surjection. 
Proof. This is Grothendieck 55, Chap. I, $\S 1$, no. 2, Prop. 3 or Treves 67 Prop. 43.9.

Theorem 1.9. Let $E_{1}$ and $E_{2}$ be locally convex spaces, and let $F_{i}$ be a vector subspace of $E_{i}$, for $i=1,2$. If $F_{1}$ or $F_{2}$ is a nuclear space then the natural linear map of $F_{1} \hat{\otimes} F_{2}$ into $E_{1} \hat{\otimes} E_{2}$ is an isomorphism of topological vector spaces.

Proof. This is Grothendieck 55, Chap. II, §3, no. 1 Prop. 10, Cor.

These two theorems may be applied to yield the following, which is stated in categorical language with a view to its cohomological applications.

Theorem 1.10. Consider the category of Fréchet nuclear spaces where the morphisms are continuous linear maps, and also the category of projectively completed tensor products of Fréchet spaces with metrizable locally convex spaces, where the morphisms are tensor products of some continuous linear map on the Fréchet factor and the identity on the second factor. Let $F$ be a metrizable locally convex space.

Then

$$
E \longrightarrow E \hat{\otimes} F
$$

and

$$
\operatorname{Lin}\left(E_{1}, E_{2}\right) \ni u \longmapsto u \hat{\otimes} 1 \in \operatorname{Lin}\left(E_{1} \hat{\otimes} F, E_{2} \hat{\otimes} F\right)
$$

defines a covariant functor from the first mentioned category to the second. This functor is exact.

If the space $F$ is nuclear, then it is not necessary that the Fréchet spaces be nuclear and the functor is still exact.

Proof. This theorem is essentially in Grothendieck 54 and is also, proven slightly differently, in Bungart $64 \mathrm{Thm}$. 5.3.

We give here for convenience a proof following Grothendieck.

Functoriality is in fact obvious from the definition; what must be shown is exactness. Consider a short exact sequence of Fréchet nuclear spaces:

$$
0 \longrightarrow A \stackrel{f}{\longrightarrow} B \stackrel{g}{\longrightarrow} C \longrightarrow 0 \quad \text { (exact) }
$$


Since $g$, being a surjection of Fréchet spaces is open, by Thm. 1.8 we have

$$
B \hat{\otimes} F \stackrel{g \hat{\otimes} 1}{\longrightarrow} C \hat{\otimes} F \longrightarrow 0 \quad(\text { exact }) .
$$

Since $f$ is open onto its image by the assumed exactness at $B$, by Thm. 1.9 we have

$$
0 \longrightarrow A \hat{\otimes} F \stackrel{f \hat{\otimes} 1}{\longrightarrow} B \hat{\otimes} F \quad \text { (exact) }
$$

Now

$$
\operatorname{im}(f \hat{\otimes} 1)=(\operatorname{im} f) \hat{\otimes} F
$$

and, denoting the closed linear hull by $[$,

$$
\begin{aligned}
\operatorname{ker}(g \hat{\otimes} 1) & =[b \otimes d \in B \hat{\otimes} F ; g(b)=0 \text { or } d=0] \\
& =(\operatorname{ker} g) \hat{\otimes} F
\end{aligned}
$$

by a simple density argument; thus

$$
\operatorname{im}(f \hat{\otimes} 1)=\operatorname{ker}(g \hat{\otimes} 1)
$$

showing exactness at $B \hat{\otimes} F$. So finally as desired we have

$$
0 \longrightarrow A \hat{\otimes} F \stackrel{f \hat{\otimes} 1}{\longrightarrow} B \hat{\otimes} F \stackrel{g \hat{\otimes} 1}{\longrightarrow} C \hat{\otimes} F \longrightarrow 0 \quad \text { (exact). }
$$

The nuclearity of a space was only used in applying Thm. 1.9, and for that it is sufficient that $F$ be nuclear. So both assertions of the theorem are proved.

\section{§2. Pure Codimensionality of $\boldsymbol{R}^{n}$ with Respect to ${ }^{E} \mathcal{O}$}

It is as a result of the pure codimensionality of $\boldsymbol{R}^{n}$ with respect to the sheaf $E_{\mathcal{O}}$ that one may define hyperfunctions and indeed that it is natural so to do. Unfortunately in order to get this result as a deduction from the general results on tensor products, we shall have to assume that $E$ is Fréchet. In order to use Thm. 1.8 and thus Thm. $1.10, E$ has to be metrizable and in order to be a good space of values 
for holomorphic functions $E$ must be quasi-complete; these two assumptions together imply $E$ is Fréchet [Treves 67 Prop. 34.3]. We first recall two generalizations of well-known results.

Theorem 2.1. (Oka-Cartan Theorem B) If $\Omega$ is a Stein submanifold of $\mathbb{C}^{n}$ and $E$ is a Fréchet space, then for $p \geqq 1$

$$
H^{p}\left(\Omega,{ }^{E} \mathcal{O}\right)=0,
$$

where $H^{p}$ denotes the ordinary cohomology with coefficients in a sheaf.

Proof. We can prove this as in the scalar case by use of the Dolbeault complex; it is a special case of Theorem 4 of Bishop 62, or of Theorem B of Bungart 64.

Theorem 2.2. (Malgrange) Let $V$ be an open set in $\mathbb{C}^{n}$ and $E$ be a Fréchet space, then for $p \geqq n$

$$
H^{p}\left(V,{ }^{E} \mathcal{O}\right)=0
$$

Proof. This follows simply, as does the previous theorem, from the fact that the Dolbeault resolution is a soft resolution of $E_{\mathcal{O}}$ and so we may calculate the cohomology from it:

$$
0 \longrightarrow{ }^{E} \mathscr{E}^{(0,0)}(V) \stackrel{\bar{\partial}}{\longrightarrow} E_{\mathscr{E}}(0,1)(V) \stackrel{\bar{\partial}}{\longrightarrow} \cdots
$$

or

$$
0 \longrightarrow \mathscr{E}^{(U, \cdot)}(V) \hat{\otimes} E .
$$

But Malgrange's Vanishing Theorem [Malgrange 57 Lemma 3] says the complex $\left(\mathscr{E}^{(0, \cdot)}(V), \bar{\partial}\right)$ is exact at $\mathscr{E}^{(0, n)}(V)$, and beyond to the right of course, so that using Thm. 1.10 , we conclude exactness at $\mathscr{E}^{(0, n)}(V) \hat{\otimes}$ E.

We can now proceed to the theorem basic to the theory of hyperfunctions, which is here being treated following Harvey and Komatsu. [Harvey 66, 69, Komatsu 66, 72]

Definition 2.3. (Sato) Let $S$ be a locally closed subset of a space 
on which there is given a sheaf $\mathscr{F}$. The subset $S$ is said to be purely $n$-codimensional with respect to $\mathscr{F}$ if

$$
\mathscr{H}^{p}(\mathscr{F})=0 \quad \text { for } \quad p \neq n .
$$

Here the $p$-th derived sheaf of $\mathscr{F}$ with support in $S$, called by Sato the sheaf of $p$-distributions on $S$ of type $\mathscr{F}$, is defined as the sheaf associated to the presheaf obtained from relative cohomology

$$
U \longrightarrow H_{U \cap S}^{p}(U, \mathscr{F})
$$

Theorem 2.4. $\boldsymbol{R}^{n}$ is purely n-codimensional with respect to the sheaf $E_{\mathcal{O}}$ over $\mathbb{C}^{n}$, that is for $p \neq n$ and $\Omega$ in a basis of the open sets of $\boldsymbol{R}^{n}$ with $n>0$,

$$
H_{\Omega}^{p}\left(V,{ }^{E} \mathcal{O}\right)=0
$$

where $V$ is a complex neighbourhood of $\Omega$, which contains $\Omega$ as a closed subset.

Proof. It is enough to show $H_{\boldsymbol{R}^{n} \cap V}^{p}\left(V,{ }^{E} \mathcal{O}\right)=0$ for $p \neq n$ and $V$ a bounded open set of $\mathbb{C}^{n}$. By the excision theorem for relative cohomology and Grauert's Neighbourhood Theorem we may choose $V$ to be Stein. Let $\Omega=\mathbb{R}^{n} \cap V$ then. By the long exact sequence of supported cohomology (Komatsu 66 Thm. 11 ii)

$$
\begin{aligned}
\cdots & \longrightarrow H_{\Omega}^{p}\left(V,{ }^{E} \mathcal{O}\right) \longrightarrow H^{p}\left(V,{ }^{E} \mathcal{O}\right) \longrightarrow H^{p}\left(V \backslash \Omega,{ }^{E} \mathcal{O}\right) \\
& \longrightarrow H_{\Omega}^{p+1}\left(V,{ }^{E} \mathcal{O}\right) \longrightarrow \cdots
\end{aligned}
$$

(exact)

If $V$ is Stein, as we have seen, $H^{p}\left(V,{ }^{E} \mathcal{O}\right)=0$ for $p>0$, so that for $p \geq 2$

$$
H_{\Omega}^{p}\left(V,{ }^{E} \mathcal{O}\right) \cong H^{p-1}\left(V \backslash \Omega,{ }^{E} \mathcal{O}\right)
$$

By the vectorized version of the Malgrange vanishing theorem, Thm. 2.2, we have for $p \geqq n+1$

$$
H_{\Omega}^{p}\left(V,{ }^{E} \mathcal{O}\right)=0
$$


For $n-1>p>0$ we already know $H^{p}(V \backslash \Omega, \mathcal{O})=0$. This is easily seen when one notes that by the usual exact sequence,

$$
\begin{aligned}
\cdots & \longrightarrow H_{\Omega}^{p}(V, \mathcal{O}) \longrightarrow H^{p}(V, \mathcal{O}) \longrightarrow H^{p}(V \backslash \Omega, \mathcal{O}) \longrightarrow \\
& \longrightarrow H_{\Omega}^{p+1}(V, \mathcal{O}) \longrightarrow \cdots
\end{aligned}
$$

(exact),

this amounts to Sato's theorem that, for $0 \leqq p<n, H_{\Omega}^{p}(V, \mathcal{O})=0$. But we may use the ordinary Dolbeault resolution

$$
0 \longrightarrow \mathcal{O} \longrightarrow \mathscr{E}^{(0, \cdot)}
$$

to calculate this cohomology; and indeed we may equally use the 'vectorized' form of this, an $E$-Dolbeault resolution to calculate $H^{p}\left(V \backslash \Omega,{ }^{E} \mathcal{O}\right)$. Now the modules in this resolution are Fréchet spaces and so tensoring by $\hat{\otimes} E$ is an exact functor. Thus $H^{p}\left(V \backslash \Omega,{ }^{E} \mathcal{O}\right)$ vanishes wherever $H^{p}(V \backslash \Omega, \mathcal{O})$ does. Thus as required, for $0<p<n-1 H^{p}\left(V \backslash \Omega,{ }^{E} \mathcal{O}\right)=0$.

This leaves the tag end of the sequence,

$$
0 \longrightarrow H_{\Omega}^{0}\left(V,{ }^{E} \mathcal{O}\right) \longrightarrow H^{0}\left(V,{ }^{E} \mathcal{O}\right) \longrightarrow H^{0}\left(V \backslash \Omega,{ }^{E} \mathcal{O}\right) \longrightarrow H_{\Omega}^{1}\left(V,{ }^{E} \mathcal{O}\right)
$$

to be dealt with. We must show the last term zero, that is

$$
\mathcal{O}(V) \hat{\otimes} E \longrightarrow \mathcal{O}(V \backslash \Omega) \hat{\otimes} E \longrightarrow 0 \quad \text { (exact) }
$$

But by the known vanishing of $H_{\Omega}^{1}(V, \mathcal{O})$ when $n \neq 1$ we have

$$
\mathcal{O}(V) \longrightarrow \mathcal{O}(V \backslash \Omega) \longrightarrow 0 \quad \text { (exact) }
$$

and since it is onto the first restriction map is an epimorphism. The desired result clearly follows by an application of Thm. 1.10 when $n>1$. The case $n=1$ follows at once from Thm. 2.1 and 2.2. Now define hyperfunctions as the only non-vanishing relative cohomology group.

Definition 2.5. Let $E$ be a Fréchet space. The space of $E$-valued hyperfunctions on an open set $\Omega$ in $\boldsymbol{R}^{n}$ is defined to be

$$
{ }^{E} \mathscr{B}(\Omega) \equiv H_{\Omega}^{n}\left(V,{ }^{E} \mathcal{O}\right)
$$


where $\mathrm{V}$ is an open set in $C^{n}$ containing $\Omega$ as a closed.

By the excision theorem [Komatsu $66 \mathrm{Thm} .1 .1, \mathrm{i}$ ] this definition does not depend on what particular complex neighbourhood $V$ of $\Omega$ is chosen. Now we can prove the analogue of Sato's theorem.

Theorem 2.6. The assignment $\Omega \rightarrow^{E} \mathscr{B}(\Omega)$ for $\Omega$ open in $\boldsymbol{R}^{n}$ and the natural restriction maps define a sheaf. This sheaf, ${ }^{E} \mathscr{B}$, is flabby.

Proof. This is an immediate consequence, as suggested in Sato $59 / 60$, of Thm. 2.4 and the facts of general sheaf theory stated in Komatsu 66 Thm. 1.8 .

As a matter of fact the Palamodov splitting result of Thm. 1.6 does give some information in the general case of quasi-complete $E$.

Proposition 2.7. If $V$ is a Stein complex neighbourhood of $\Omega$, and $p \geqq 2$ then

$$
H_{\Omega}^{p}\left(V,{ }^{E} \mathcal{O}\right) \cong H^{p-1}\left(V \backslash \Omega,{ }^{E} \mathcal{O}\right)
$$

Proof. This is an immediate consequence of Thm. 1.6 and the canonical long exact sequence of relative cohomology

$$
\begin{aligned}
\cdots & \longrightarrow H_{\Omega}^{p}\left(V,{ }^{E} \mathcal{O}\right) \longrightarrow H^{p}\left(V,{ }^{E} \mathcal{O}\right) \longrightarrow H^{p}\left(V \backslash \Omega,{ }^{E} \mathcal{O}\right) \\
& \longrightarrow H_{\Omega}^{p+1}\left(V,{ }^{E} \mathcal{O}\right) \longrightarrow \cdots
\end{aligned}
$$

(exact).

\section{§3. Hyperfunctions as Boundary Values}

Because of the analogue of the Oka-Cartan theorem, Thm. 2.1, $E$-valued hyperfunctions may be viewed as boundary values of holomorphic functions in the usual way. Let $\left(\mathscr{V}, \mathscr{V}^{\prime}\right)$ be a Stein relative covering of $(V, V \backslash \Omega)$ where $\Omega$ is an open set of $\boldsymbol{R}^{n}$ and $V$ is a complex neighbourhood of it. (Recall that this means $V$ is an open set of $\mathbb{C}^{n}$ containing $\Omega$ as a closed subset). Then since finite intersections of Stein open sets are still Stein sets, this relative covering is acyclic and

$$
{ }^{E} \mathscr{B}(\Omega) \cong H^{n}\left(\mathscr{V}, \mathscr{V}^{\prime} ;{ }^{E} \mathcal{O}\right)
$$


To choose one specific and useful relative covering, recollect that by Grauert's Theorem there is an arbitrarily small Stein neighbourhood $V$ of $\Omega$ such that $V \cap \boldsymbol{R}^{n}=\Omega$ [Grauert 58 \$3.4]. Then let

$$
\text { and } \quad \begin{aligned}
V_{0} & =V \\
V_{j} & =\left\{z \in V ; \operatorname{Im} z_{j} \neq 0\right\}, \quad j=1, \ldots, n \\
\mathscr{V}^{\prime} & =\left\{V_{1}, \ldots, V_{n}\right\} \\
\mathscr{V} & =\mathscr{V}^{\prime} \cup\left\{V_{0}\right\} .
\end{aligned}
$$

All these sets $V_{j}$ are Stein so

$$
\begin{aligned}
{ }^{E} \mathscr{B}(\Omega) & \cong H^{n}\left(\mathscr{V}, \mathscr{V}^{\prime} ;{ }^{E} \mathcal{O}\right) \\
& \cong{ }^{E} \mathcal{O}(V \# \Omega) / \Sigma_{j}{ }^{E} \mathcal{O}\left(\widehat{V}_{j}\right)
\end{aligned}
$$

where

$$
\begin{aligned}
V \# \Omega & =\left\{z \in V ; j=1, \ldots, n \Rightarrow \operatorname{Im} z_{j} \neq 0\right\} \\
\hat{V}_{j} & =\left\{z \in V ; k \neq j \Rightarrow \operatorname{Im} z_{k} \neq 0\right\} .
\end{aligned}
$$

For any function $f$ in ${ }^{E} \mathcal{O}(V \# \Omega)$ the associated cohomology class is denoted $[f]$, and then $f$ is termed a defining function of the hyperfunction $[f]$.

Near $\boldsymbol{R}^{n}, V \# \Omega$ has $2^{n}$ connected components corresponding to the possible signs of the imaginary parts of $z$; they are $V \cap\left(\boldsymbol{R}^{n}+i \Gamma_{\sigma}\right)$ where $\Gamma_{\sigma}=\left\{y \in \boldsymbol{R}^{n} ; \forall j=1, \ldots, n \sigma_{j} y_{j}>0\right\}$ and $\sigma: j \mapsto \sigma_{j}:(1, \ldots, n) \rightarrow\{+1,-1\}$ runs over all such $n$-tuples of signs. Intuitively speaking, the hyperfunction $[f]$ is the sum of boundary values

$$
[f](x)=\Sigma_{\sigma}\left(\prod_{j=1}^{n} \sigma_{j}\right) f\left(x+i \Gamma_{\sigma} 0\right) .
$$

Of course the exact meaning of such boundary values has not been made explicit here, see Komatsu 72 for further discussion. See also Ehrenpreis 61, 70 and Martineau 64 for related topics.

Other coverings may be of more use on particular occasions.

Looking at it from the reverse point of view, giving a collection of $2^{n} E$-valued functions each holomorphic in one of the tubes $\boldsymbol{R}^{n}+i \Gamma_{\sigma}$, that is in ${ }^{E} \mathcal{O}\left(\boldsymbol{R}^{n}+i \Gamma_{\sigma}\right)$, defines a class of the cohomology $H^{n}\left(\mathscr{V}, \mathscr{V}^{\prime} ;{ }^{E} \mathcal{O}\right)$ where 


$$
\begin{aligned}
& \mathscr{V}^{\prime}=\left\{\boldsymbol{R}^{n}+\sqrt{-1} H_{j} ; j=1, \ldots, n\right\}, \\
& \mathscr{V}=\mathscr{V}^{\prime} \cup\left\{\boldsymbol{C}^{n}\right\},
\end{aligned}
$$

and $H_{j}=\left\{y \mid y_{j}>0\right\}$ is a half-space. Thus such a collection of functions defines a hyperfunction.

\section{§4. Hyperfunctions with Holomorphic Parameters}

As should be obvious to the reader most of the Harvey-Komatsu development of Sato's theory of hyperfunctions generalizes to the case of values in a Fréchet space $E$. For instance all the statements and proofs from page 25 up to and including page 31 of Komatsu 72 continue to hold true with the addition of a presuperscript $E$ to the sheaves $\mathcal{O}$ and $\mathscr{r}^{n-r} \mathcal{O}$ and with the usual interpretation.

As an example we set out part of the proof of the following theorem which provides an alternative demonstration of the pure $n$-codimensionality of $\boldsymbol{R}^{n}$ in $\boldsymbol{C}^{n}$ with respect to ${ }^{E} \mathcal{O}$.

Take the generic point of $\boldsymbol{C}^{n}$ to be $z=\left(z_{1}, \ldots, z_{n}\right)$.

Let

$$
R_{j}=\left\{z \in \boldsymbol{C}^{n} ; z_{j} \in \boldsymbol{R}\right\} \quad \text { for } \quad j=1, \ldots, n
$$

and let

$$
R(\phi)=C^{n}, \quad \text { and for } \quad \phi \neq J \subset\{1, \ldots, n\}
$$

define

$$
R(J)=\cap\left\{R_{j} ; j \in J\right\} .
$$

Theorem 4.1. $R(J)$ is purely r-codimensional with respect to the sheaf ${ }^{E} \mathcal{O}$ on $C^{n}$, where $r$ is the cardinality of $J$.

Write ${ }^{E} \mathscr{B} J \mathcal{O} \equiv \mathscr{H}_{R(J)}^{r}\left({ }^{E} \mathcal{O}\right)$

Then for $p>0$ and every Stein open set $V$ in $\mathbb{C}^{n}$

$$
H^{p}\left(V,{ }^{E} \mathscr{B} J \mathcal{O}\right)=0
$$

Proof. Heuristically one should view the sheaf ${ }^{E} \mathscr{B} J \mathcal{O}$ as that of germs of functions which are hyperfunctional in the $J$ variables and holomorphic in the rest.

If $\mathrm{r}=0$, so $J=\phi$ and $R(\phi)=\mathbb{C}^{n}$, the assertion is Thm. 2.1. 
We shall prove the theorem by induction on $r$. It is clearly possible without loss of generality, by relabelling of variables if necessary, to take $J=J_{r}=\{1, \ldots, r\}$ for $r \geqq 1$; and in this case let us abbreviate by $R(1,2, \ldots, r) \equiv R(r)=\boldsymbol{R}^{r} \times \mathbb{C}^{n-r}$. The start of the induction step is contained in the following lemma.

Lemma 4.2. If Theorem 4.1 holds for some $r$ and for all Stein open sets $V$ in $\boldsymbol{C}^{n}$

$$
H_{R_{r+1} \cap V}^{0}\left(V,{ }^{E} \mathscr{B} J_{r} \mathcal{O}\right)=0,
$$

then Theorem 4.1 holds for $(r+1)$ and for all such $V$ we have

$$
{ }^{E} \mathscr{B} J_{r+1} \mathcal{O}(V)={ }^{E} \mathscr{B} J_{r} \mathcal{O}\left(V \backslash R_{r+1}\right) /{ }^{E} \mathscr{B} J_{r} \mathcal{O}(V)
$$

Proof. For the sake of ease of writing let us set ${ }^{E} \mathscr{B} J_{r} \mathcal{O}={ }^{E} \mathscr{B}_{r}$. Let $V$ be a Stein open set in $\mathbb{C}^{n}$, then for $p \neq 1$

$$
H_{R_{r+1} \cap V}^{p}\left(V,{ }^{E} \mathscr{B}_{r}\right)=0
$$

For $p=0$ this is an assumption. For $p \geqq 2$ it will follow from the exact sequence of relative cohomology

$$
\begin{aligned}
\cdots \longrightarrow & H_{R_{r+1}}^{p}\left(V,{ }^{E} \mathscr{B}_{r}\right) \longrightarrow H^{p}\left(V,{ }^{E} \mathscr{B}_{r}\right) \longrightarrow H^{p}\left(V \backslash R_{r+1},{ }^{E} \mathscr{B}_{r}\right) \\
& \longrightarrow H_{R_{r+1}}^{p+1}\left(V,{ }^{E} \mathscr{B}_{r}\right) \longrightarrow \cdots
\end{aligned}
$$

(exact)

For $p \geqq 1$ since $V$ and $V \backslash R_{r+1}$ are both Stein, by assumption

$$
H^{p}\left(V,{ }^{E} \mathscr{B}_{r}\right)=0=H^{p}\left(V \backslash R_{r+1},{ }^{E} \mathscr{B}_{r}\right) .
$$

Now ${ }^{E} \mathscr{B}_{\boldsymbol{r}}$ is by definition a sheaf with support in the closed set $R(r)$ so

$$
H_{R_{r+1} \cap V}^{p}\left(V,{ }^{E} \mathscr{B}_{r}\right)=H_{R(r+1) \cap V}^{p}\left(V,{ }^{E} \mathscr{B}_{r}\right) .
$$

Since a point of $\boldsymbol{C}^{n}$ has a neighbourhood base consisting of Stein open sets it follows that $R(r+1)$ is purely 1-codimensional with respect to ${ }^{E} \mathscr{B}_{r}$. By a result of general sheaf theory [Komatsu $66 \mathrm{Thm} .1 .9$ ] 
we have that $R(r+1)$ is purely $(r+1)$-codimensional with respect to ${ }^{E_{\mathcal{O}}}$. Thus we have the first assertion of Thm. 4.1 for $(r+1)$. There are also the isomorphisms [Komatsu 66 Thm. 1.8]

$$
\begin{aligned}
H^{p}\left(V,{ }^{E} \mathscr{B}_{r+1}\right) & \cong H^{p}\left(V, \mathscr{H}_{R(r+1)}^{1}\left({ }^{E} \mathscr{B}_{r}\right)\right) \\
& \cong H_{R(r+1) \cap V}^{p+1}\left(V,{ }^{E} \mathscr{B}_{r}\right) \\
& \cong H_{R_{r+1}+1}^{p+1}\left(V,{ }^{E} \mathscr{B}_{r}\right)
\end{aligned}
$$

and using these the rest of Thm. 4.1 for $(r+1)$ follows as does the last assertion of Lemma 4.2.

The induction can proceed if the additional assumption invoked in lemma 4.2 can be shown to be generally true.

This assumption can however be proved in completely the same way as is done for the scalar case in Komatsu 72 pp. 29-31, if use is made of the Hartogs' theorem for $E$-valued holomorphic functions, see Komatsu 72 for details.

The arguments just given have a different tone from that of earlier sections, in that they are in a sense more elementary. The method also gives an elementary proof of the results of section 2, and conversely the arguments of section 2 may be used to give the results of this section.

\section{References}

[1] Bishop, E., Analytic functions with values in a Fréchet space, Pacific J. Math., 12 (1962), 1177-1192.

[2] Bungart, L., Holomorphic functions with values in locally convex spaces and applications to integral formulas, Trans. Amer. Math. Soc., $\mathbb{1} 1 \mathbb{1}$ (1964), 317-344.

[3] Ehrenpreis, L., Analytically uniform spaces and some applications, Trans. Amer. Math. Soc., 101 (1961), 52-74.

[4] - Fourier Analysis in Several Complex Variables, Wiley-Interscience, New York/London et al., 1970.

[5] Grauert, H., On Levi's problem and the imbedding of real analytic manifolds, Ann. of Math., (2) 68 (1958), 460-472.

[6] Grothendieck, A., Sur certains espaces de fonctions holomorphes I, II, Jour. reine angew. Math., 192 (1953), 35-60, 77-95.

[7] - Opérations algébriques sur les distributions à valeurs vectoriellesThéorème de Künneth., Sém. Schwartz 1953/54, exp. 24, 16/6/54, (1954). 
[8] — Produits Tensoriels Topologiques et Espaces Nucléaires, Mem. Amer. Math. Soc., Providence, R. I., 16 (1955).

[9] Gunning, R. C. and Rossi, H., Analytic Funclions of Several Complex Variables, Prentice Hall Inc., Englewood Cliffs, N. J., 1965.

[10] Harvey, F. R., Hyperfunctions and linear partial differential equations, Stanford U. Thesis, 1966.

[11] The theory of hyperfunctions on totally real subsets of a complex manifold with applications to extension problems, Amer. J. Math., 91 (1969), 853-873.

[12] Hörmander, L., An Introduction to Complex Analysis in Several Variables, Van Nostrand, Princeton, N. J., 1966.

[13] Komatsu, H., Relative cohomology of sheaves of solutions of differential equations, Lecture Notes in Math., Springer-Verlag, Berlin/Heidelberg/New York, 287 (1972), 192-261, (first publ. Sém. Lions-Schwartz 1966).

[14] - An introduction to the theory of hyperfunctions, Lecture Notes in Math., Springer-Verlag, Berlin/Heidelberg/New York, 287 (1972), 3-40.

[15] Malgrange, B., Faisceaux sur les variétés analytiques réelles, Bull. Soc. Math. France, 85 (1957), 231-237.

[16] Martineau, A., Distributions et valeurs au bord des functions holomorphes, Proc. Intern. Summer School on the Theory of Distributions, Lisbon, (1964) 195-326.

[17] Ouchi, S., On abstract Cauchy problems in the sense of hyperfunctions, Lecture Notes in Math., Springer-Verlag, Berlin/Heidelberg/New York, 287 (1972), 135-152.

[18] Palamodov, V.P., On a Stein manifold the Dolbeault complex splits in positive dimensions, Math. U.S.S.R. Sbornik, 17 (1972), 289-316 (transl. of Mat. Sbornik, 88 (130) No. 2 (1972), 287-315).

[19] Sato, M., Theory of hyperfunctions I and II, J. Fac. Sci., Univ. Tôkyô, Sect. I, 8 (1959/60), 139-193, 387-436.

[20] Schwartz, L., Espaces de fonctions différentiables à valeurs vectorielles, $J$. Analyse Math., 4 (1952), 88-148.

[21] - Distributions à valeurs vectorielles, Chap. I, Ann. Inst. Fourier, Grenoble, 7 (1957), 1-141.

[22] — Distributions à valeurs vectorielles, Chap. II, Ann. Inst. Fourier, Grenoble, 8 (1958), 1-209.

[23] Treves, F., Topological Vector Spaces, Distributions and Kernels, Academic Press, London/New York, 1967. 
